\title{
The McCollough effect: Dissociating retinal from spatial coordinates
}

\author{
FELICE L. BEDFORD and KAREN S. REINKE \\ University of Arizona, Tucson, Arizona
}

\begin{abstract}
Three experiments were conducted to dissociate the perceived orientation of a stimulus from its orientation on the retina while inducing the McCollough effect. In the first experiment, the typical contingency between color and retinal orientation was eliminated by having subjects tilt their head $90^{\circ}$ for half of the induction trials while the stimuli remained the same. The only relation remaining was that between color and the perceived or spatial orientation, which led to only a small contingent aftereffect. In contrast, when the spatial contingency was eliminated in the second experiment, the aftereffect was as large as when both contingencies were present. Finally, a third experiment determined that part of the small spatial effect obtained in the first experiment could be traced to hidden higher order retinal contingencies. The study suggested that even under optimal conditions the McCollough effect is not concerned with real-world properties of objects or events. Implications for several classes of theories are discussed.
\end{abstract}

The McCollough effect (ME) is viewed by some researchers to be an instance of learning; the most well developed explanation in that class has appealed to Pavlovian conditioning (e.g., Allan \& Siegel, 1986; Murch, 1976; Siegel, Allan, \& Eissenberg, 1992; Westbrook \& Harrison, 1984). For others, the phenomenon appears closer to sensory fatigue than to learning (e.g., Harris \& Gibson, 1968; McCollough, 1965; Stromeyer, 1978). To produce the effect, a vertical grid consisting of alternating magenta and black lines may be alternated with a horizontal grid of green and black lines. Following a few minutes of repeated stimulation, color becomes contingent on orientation such that achromatic vertical lines appear a weakly saturated green and horizontal lines appear pink.

Learning models emphasize forging new connections between previously unrelated entities, in this instance, vertical lines and red color. Specific Pavlovian interpretations suggest that vertical lines serve as the conditioned stimulus and red color as the unconditioned stimulus. " $\mathrm{Fa}$ tigue models" usually assume that the linkages between orientation and color already exist prior to induction, which seems to leave little for learning to do. For instance, Gibson and Harris (1968, see Harris, 1980) proposed adaptation within detectors jointly sensitive to orientation and color. In addition, the models typically propose that illusory colors result from decreased sensitivity to prolonged stimulation, an outcome that is usually thought of as fatigue rather than learning.

Several questions are thought relevant to distinguishing between these two general approaches. For instance,

We would like to thank Celeste McCollough Howard for generous use of time and equipment to calibrate our computer monitor and for helpful comments. This article is dedicated to Viking. We also thank two other (anonymous) reviewers for helpful comments. Correspondence should be addressed to F. L. Bedford, Department of Psychology, University of Arizona, Tucson, AZ 85721 (e-mail: bedford@ccit.arizona.edu). how many pairs of attributes other than color and oriented lines can lead to contingent aftereffects (see Dodwell \& Humphrey, 1990; Harris, 1980; Siegel et al. 1992; Skowbo, 1984; Stromeyer, 1978)? Color can be made contingent on velocity, direction of motion can be made contingent on intensity, and orientation can be made contingent on spatial frequency, to name a few (Mayhew \& Anstis, 1972; Stromeyer \& Mansfield, 1970). Because learning models tend to emphasize establishing new connections, a large number of different contingent aftereffects should be possible. On the other hand, fatigue models predict that relatively few stimuli will be effective, because there must be a limit to the number of preexisting detectors sensitive to arbitrary pairs of features. The data appear to fall between the two extremes.

A second issue debated recently concerns whether stimuli consisting of only homogeneous color without the oriented lines will diminish the ME. According to Pavlovian models, interspersing these trials among the usual induction stimuli should decrease the contingency relation, because color appears in the absence of orientation as well as in its presence. This in turn should lead to a smaller aftereffect (Skowbo, 1984; Skowbo \& Forster, 1983). Fatigue models predict no particular influence from the added trials. Some induction manipulations that present the color without orientation are influential (Siegel et al., 1992), and others are not (Siegel \& Allan, 1987; Siegel, Allan, Roberts, \& Eissenberg, 1990; Skowbo \& Forster, 1983).

One potentially relevant factor that has received less attention concerns whether the color becomes contingent on retinal or on spatial orientation. ${ }^{1}$ McCollough (1965) found that when an observer tilted his or her head $90^{\circ}$ following induction, the colors on the test patterns reversed positions. For instance, on some trials the test patterns consisted of vertical lines on the left and horizontal 
lines on the right. If the vertical lines appeared pink and the horizontal lines appeared green, tilting the head caused the vertical lines to instead appear green and the horizontal lines pink. Because the orientation perceived by an observer remains unchanged with head tilt (vertical lines continue to appear vertical when tilted), the finding suggested that it is the retinal orientation that is critical.

In subsequent work, Ellis (1976) has confirmed the finding in more quantitative detail, Thompson and Latchford (1986) have found also that it is the retinal rather than the apparent "color" that determines the ME, and Harris $(1970,1980)$ has demonstrated generality to at least one other contingent aftereffect. In the last study, color was made contingent on spatial frequency by pairing green with wide (vertical) bars and red with narrow (vertical) bars. Testing revealed that it was the retinal and not the apparent spatial frequency that controlled the illusory colors. Details of the studies, as well as two contradictory reports (Jordan \& Uhlarik, 1983; Mikaelian, 1976), will be examined in the General Discussion. Harris (1980, p. 134) suggests that "this dependence on retinal geometry is a serious problem for a learning model, since learning normally depends on objects as perceived rather than on details of the proximal stimulus pattern." Should the overall findings be viewed as compelling evidence against learning interpretations?

The answer to that question may depend not just on whether the ME is dependent on spatial properties but on whether it can be made dependent on spatial properties. The powerful demonstration whereby colors appear to reverse following head tilt does dissociate retinal from spatial coordinates, but does so during testing only. That is, during training there exists both a retinal and a spatial contingency. Because the head is always upright, color is paired with both retinal and spatial orientation. Logically, acquiring either dependence will correctly reflect the contingencies of the environment. When the system is given the option, it is clear that the retinal properties dominate. However, it is not known what will happen if that contingency is removed, thereby leaving only a spatial contingency. Such a situation may even be closer to learning in the real world, where constant motion of an observer would prevent any long-term retinal correlations. The empirical goal of the present study was to investigate McCollough-like effects while dissociating the two coordinates during training.

\section{EXPERIMENT 1}

The goal of Experiment 1 was to determine if color could be made contingent on spatial orientation once the retinal contingency was eliminated. This was accomplished by having each subject tilt her head $90^{\circ}$ for part of the induction period. For instance, imagine standard stimuli where a red grating that is objectively vertical alternates with a green grating, objectively horizontal. If viewed when upright, red is paired with both spatial and retinal vertical, and green is paired with both spatial and retinal horizontal. If the same stimuli are next viewed with the head tilted, red remains paired with spatial vertical and green remains paired with spatial horizontal, but the retinal relation has reversed. Red is now paired with retinal horizontal, and green with retinal vertical. Changing the relative proportion of trials between head upright and head tilted allows explicit control over the retinal contingency. Equal numbers of the two types of trials produce zero contingency; both horizontal and vertical lines on the retina will each be red half the time and green the other half.

Such a spatial-only condition was compared with a more standard induction where both contingencies co-exist, except with head position always at $90^{\circ}$ instead of always upright. This sets an upper limit on expected contingent aftereffects to which the magnitude of the other group could be compared.

\section{Method}

Subjects. The subjects were 16 female undergraduates participating for course credit in introductory psychology at the University of Arizona.

Apparatus and Materials. Two headrests were constructed on top of adjustable stands, one horizontally oriented for the $90^{\circ}$ head position and one vertically oriented for the upright head position. The top of the vertical headrest was set to a height of 47.5 in. and placed to the left of the subject; the top of horizontal headrest was set to 35.5 in. and placed on the right. The chair height was adjusted for each subject such that the eyes were 46 in. from the floor while in the upright position.

The stimuli were presented on a Zenith Data Systems VGA flat screen monitor (Model ZCM-1490) driven by an IBM AT-compatible computer equipped with a Paradise VGA Plus Card. The system allowed $256 \mathrm{~K}$ different colors where up to 16 of them could be displayed at once, at $640 \times 480$ resolution. Individual colors were selected by combining red, green, and blue (R, G, and B) values, each of which allowed 6 bits of information. The values ranged therefore from 0 to $63\left(2^{6}\right)$ for each color. White was seen when each color was at maximum $(63,63,63)$, black when each was at minimum $(0,0,0)$, and gray in between (e.g., 22, 22, 22). Chromatic colors were created by changing the relative proportions of the three numbers (see, e.g., Siegal \& Allan, 1987). The monitor was located 29 in. from the subject.

The colors of the two induction stimuli were green $(0,63,0)$ and magenta $(63,0,34)$ (see Table 1$)$. The test colors were constrained to vary along a green-to-red continuum, ranging from maximally saturated green $(0,63,0)$ to a central point of white $(63$, $63,63)$ to maximally saturated red $(63,0,0)$. Greens of intermediate saturations were created by keeping the value for green at maximum, while simultaneously decreasing the values for blue and red, and for reds by keeping the value for red at maximum while simultaneously decreasing the values for blue and green. For instance, one unit of green would be $(62,63,62), 2$ units of green $=(61$, $63,61)$, and so on. One unit of red $=(63,62,62)$, and so on. The test range used by the subjects varied from 0 to 8 units for both red and green. A single keypress (" $G$ ") added one unit of green, and each press of another key (" $\mathrm{H}$ ") added a unit of red. Color could be changed quickly by holding down the appropriate key.

Calibration. A radiometer (Photo Research PR 703A-PC) was used to measure the chromaticity of the monitor as a function of voltage change to the RGB guns. Table 1 shows the conversion for the induction stimuli and test color range used in the experiment to CIE $1976\left(u^{\prime} v^{\prime}\right)$ uniform-chromaticity-scale (UCS) coordinates. Equal displacements in UCS space is a closer approximation to perceptually equal color differences than $x, y$ space (see, e.g., Wyszecki, 
Table 1

Conversion From Monitor Color to $u^{\prime}$ and $v^{\prime}$

\begin{tabular}{lcccc}
\hline & RGB & $u^{\prime}$ & $v^{\prime}$ & Luminance $\left(\mathrm{cd} / \mathrm{m}^{2}\right)$ \\
\hline Magenta Induction & $(63,0,34)$ & .3415 & .4401 & 25.76 \\
Green Induction & $(0,63,0)$ & .1346 & .5613 & 80.10 \\
Red Test Range & $(63,63,63)$ to $(63,55,55)^{*}$ & $.1913+.0016 X \dagger$ & $.4650+.0005 X \ddagger$ & 130.8 to 103.8 \\
Green Test Range & $(63,63,63)$ to $(55,63,55)^{*}$ & $.1920-.0013 X \dagger$ & $.4651+.0020 X \dagger$ & 130.8 to 119.5 \\
\hline
\end{tabular}

Note-Correlations for best fit lines were based on an average of three measurements at each of the 8 points. Most of the luminance changes in the red test range occurred from white to 1 unit (130.8 to 122.2) and from 6 units to 7 units (113.1 to 103.8). ${ }^{* 0-8}$ units. $\nmid r^{2}=.99 . \quad \ddagger r^{2}=.92$.

1986). Over the range of test colors used, each additional unit produced a linear step in $\boldsymbol{u}^{\prime} v^{\prime}$ space for both red and green. Consequently, output of the computer guns was a reasonable estimate of color strength in color space and was used throughout to measure strength of the aftereffects (e.g., Day, Webster, Gillies, \& Crassini, 1992; Webster, Day, \& Willenberg, 1988). Figure 1a shows the induction and other stimuli in the UCS diagram; Figure $1 \mathrm{~b}$ magnifies the test range used in the experiment (red, $v^{\prime}=.40+.32 u^{\prime}$, $r^{2}=.91$; green, $v^{\prime}=.77-1.57 u^{\prime}, r^{2}=.99$ ).

Procedure. The subjects were told that they were participating in an experiment on color vision. They were randomly assigned to one of two groups: the spatial-only group or the both-control group. There were three main stages: initial screening and testing, induction, and test.

Initial screening and testing. The procedure used for all testing involved color matching on the computer. The subjects were able to perform matches quickly, they were satisfied with their judgments, and the measure was sufficiently sensitive for our purposes. The purpose of this stage was to give the subjects practice with color matching on the computer and practice with head tilt and to screen out subjects who could not match colors appropriately. The trials were not intended to provide a quantitative baseline from which to subtract postinduction data.

The subjects were shown a white "color circle," 1.5 in. in diameter, that could be changed to different shades of red and green using the keyboard. Following familiarization, they were given a color-matching task consisting of four trials, two with head upright and two with head tilted. Each stimulus was a square patch $(6.75 \times$ $6.75 \mathrm{in}$.) divided into quadrants: the upper left and lower right quadrants were composed of alternating black and colored vertical bars, and the upper right and lower left were composed of horizontal bars. The color circle appeared in the middle of the display. To minimize any interference from these preinduction displays, the spatial frequency of the bars $(0.64$ cycles $/ \mathrm{deg})$ was chosen to be less than half the spatial frequency to be used during induction. On each trial, an arrow pointing at one or more quadrants indicated which color the subject should match. Arrows were chosen instead of verbal indicators (such as "match to the vertical") due to the ambiguity of those names when tilted. When the match was complete, the subject struck a key (" $S$ ") to terminate the trial. The colors and head positions on the 4 trials, which was the same for all subjects, were (1) match to upper left and lower right (vertical), which was a dark pink $(31,31,63)$, while in upright position, (2) match to only the lower left (horizontal), which was white, while tilted, (3) match to upper right and lower left (horizontal), which was a lighter pink (40, 40,63 ), while upright, and (4) match to upper left and lower right (vertical), which was a light green $(40,63,40)$, while tilted. The distractor quadrants, which were not to be matched, changed color on each trial and included shades of green, red, yellow, and white.

A criterion was established such that the average difference between a subject's match and the actual color (averaged over all four trials) had to be no more than 4 units in order to continue with the experiment. ${ }^{2}$ One subject was eliminated and replaced for failing to meet the criterion.
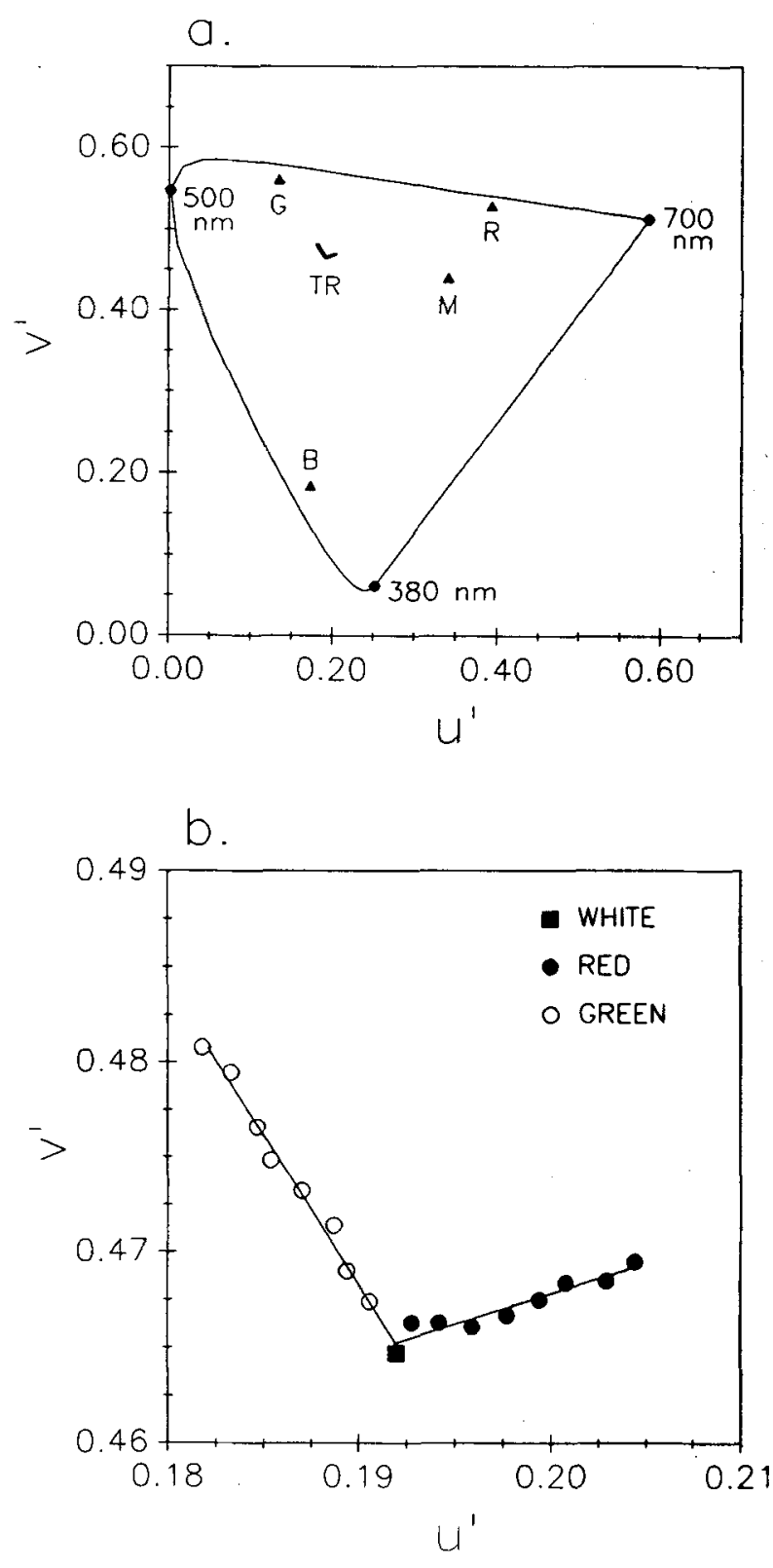

Figure 1. Computer monitor colors in CIE $1976\left(u^{\prime} v^{\prime}\right)$ UCS diagrams. Panel a: $M=$ magenta induction. $G=$ green induction and maximum value of the green gun. $R=$ maximum value of the red gun. $B=$ maximum value of the blue gun. $T R=$ test range. Panel $b$ : The range of test colors used in the experiments shown magnified. 
Induction. The subjects in both groups thus far received identical treatment. In addition, the physical displays during induction were identical for the two groups: half of each group were shown red vertical lines alternating with green horizontal lines (subgroup RVGH), and the other half received the opposite color-orientation pairing (GVRH). In the spatial-only group, the subject saw the first two stimuli (e.g., red vertical lines then green horizontal lines) with head upright, the next two repetitions with head tilted $90^{\circ}$, the two after that while upright, and so forth until the induction was complete. The induction conditions of one subgroup for all the experiments are shown in Table 2.

In the both-control group, the subjects saw all the displays while tilted $90^{\circ}$, thus receiving an analog to the standard ME but with head tilted. To control for any effects of head motion per se in the spatial-only group, each subject in the both-control group moved her head halfway to upright between trials but always returned to the tilted head position. Both the retinal and the spatial orientation varied systematically with color, and both contingencies predict that the same aftereffect colors would be seen when tested in the induction position. For instance, if subjects receive objectively red vertical and green horizontal pairs while in the tilted position, on the retina they receive red horizontal and green vertical. If color becomes contingent on spatial orientation, then objectively vertical test lines will appear green while in the tilted position. Likewise, if color becomes contingent on retinal orientation, then objectively vertical lines (horizontal on the retina) will also appear green while in the tilted position. As in the standard ME, opposite predictions require testing in a novel head position.

It was critical that each subject's head be in the correct position before a display was presented. The subjects were informed that they would be alternating between head positions (or moving and returning to the same place for the control group) after every two displays. After a pair of displays, a computer-generated beep signaled the subjects to change positions. When they had come to rest in the new position, they pushed one of two buttons on a joystick held in the hand. The button on top of the vertical joystick indicated they were upright, and a button on the right side indicated they were tilted to the right. If they were not in position after $2 \mathrm{sec}$, a series of short high-frequency beeps reminded them again to do so. A correct buttonpush was followed by the next stimulus (if a total of $2 \mathrm{sec}$ had elapsed). An incorrect buttonpush prompted instead a set of instructions on the screen indicating the correct head position. All subjects were told of the importance of head position and were reminded throughout the experiment. As a final check on head position, the experimenter watched each subject during the entire induction phase.

Table 2

Conditions of Experiments 1-3

\begin{tabular}{lclll}
\hline \multicolumn{1}{c}{ Contingency } & $\begin{array}{c}\text { Head } \\
\text { Position }\end{array}$ & \multicolumn{1}{c}{$\begin{array}{c}\text { Physical } \\
\text { (Spatial) }\end{array}$} & \multicolumn{1}{c}{ Retinal } \\
\hline Spatial only & $\mathrm{U}$ & $\mathrm{RV} / \mathrm{GH}$ & $\mathrm{RV} / \mathrm{GH}$ \\
& $\mathrm{T}$ & $\mathrm{RV} / \mathrm{GH}$ & $\mathrm{RH} / \mathrm{GV}$ \\
Both spatial and retinal & $\mathrm{T}$ & $\mathrm{RV} / \mathrm{GH}$ & $\mathrm{RH} / \mathrm{GV}$ \\
& $\mathrm{T}$ & $\mathrm{RV} / \mathrm{GH}$ & $\mathrm{RH} / \mathrm{GV}$ \\
Retinal only & $\mathrm{U}$ & $\mathrm{RV} / \mathrm{GH}$ & $\mathrm{RV} / \mathrm{GH}$ \\
& $\mathrm{T}$ & $\mathrm{RH} / \mathrm{GV}$ & $\mathrm{RV} / \mathrm{GH}$ \\
Neither & $\mathrm{U}$ & $\mathrm{RV} / \mathrm{RH}$ & $\mathrm{RV} / \mathrm{RH}$ \\
& $\mathrm{T}$ & $\mathrm{GH} / \mathrm{GV}$ & $\mathrm{GV} / \mathrm{GH}$ \\
Four-stimuli simulation & $\mathrm{U}$ & $\mathrm{RV} / \mathrm{GH}$ & $\mathrm{RV} / \mathrm{GH}$ \\
& $\mathrm{U}$ & $\mathrm{R} 105^{\circ} / \mathrm{G} 15^{\circ}$ & $\mathrm{R} 105^{\circ} / \mathrm{G} 15^{\circ}$ \\
Two-stimuli control & $\mathrm{U}$ & $\mathrm{Blank}$ & $\mathrm{Blank}$ \\
& $\mathrm{U}$ & $\mathrm{R} 105^{\circ} / \mathrm{G} 15^{\circ}$ & $\mathrm{R} 105^{\circ} / \mathrm{G} 15^{\circ}$ \\
\hline
\end{tabular}

Note--Only one subgroup is shown in the table. Actual experiments counterbalanced direction of color-orientation pairs. $\mathbf{R}=$ red, $\mathbf{G}=$ green, $\mathrm{V}=$ vertical, $\mathrm{H}=$ horizontal, $\mathrm{T}=$ tilted, $\mathrm{U}=$ upright.
Each display was on the screen for $4.0 \mathrm{sec}$, with a 2.4 -sec interstimulus interval (ISD) between the first and second display of a pair. A longer (2.9-sec) ISI was used between pairs of displays to allow sufficient time for the head movements. The screen was blank during the ISIs. There were 62 pairs of displays presented, for a total of $8 \mathrm{~min} 16 \mathrm{sec}$ of actual induction. Pilot work suggested that 62 head tilts was close to the upper limit of changing head positions without discomfort. Relatively short stimulus durations were chosen to minimize any spurious correlations that could result from the first couple of trials. The subjects were instructed to allow their eyes to wander about each pattern and not to fixate one spot on the monitor. Halfway through the induction phase, there was a 1 min break, during which time the screen was white and the subjects were allowed to stretch. The displays were $6.25 \times 6.25$ in square, and the spatial frequency of the bars was $1.66 \mathrm{cycles} / \mathrm{deg}$.

Test. Following a 60 -sec period during which the screen was white, the subjects were given a color-matching task similar to that used in the initial screening except that the spatial frequency of the test bars was identical to that of the induction bars. In addition, the nonblack bars of all the quadrants were physically white. There were 8 test displays, 4 of which had arrows pointing to both vertical line quadrants and 4 to both horizontal line quadrants. The order of the displays was 2 vertical matches, 2 horizontal matches, 2 vertical, and 2 horizontal for half of the subjects ("line testing order"), and the reverse for the other half. All subjects were tilted for the first trial and subsequently alternated between head positions after every trial for the remainder of the trials. Thus, there were two repetitions for each of 4 types of trials (vertical tilted, vertical upright, horizontal tilted, and horizontal upright). The aftereffect was measured by calculating the difference between each setting and white $(63,63,63)$, which is what the bars should appear if there were no color aftereffect. Pink (e.g., 63, 60, 60) was scored arbitrarily as negative ( -3 , in this example), and green (e.g., 60 , $63,60)$ was scored as positive $(+3$, in this example). The greater the difference, the stronger the color that was seen. The subjects were asked a few questions at the end of the experiment, including whether or not they noticed any pattern to the induction stimuli.

\section{Results and Discussion}

As expected, subjects in the both-control group exhibited the ME even though induction occurred with a tilted head position. Strength of the effect in that group can be seen in the right panel of Figure 2. The data are shown collapsed across repetition and the direction of colororientation pairs received during induction: Subjects receiving objectively red-vertical/green-horizontal (RVGH) were averaged with those receiving red-horizontal/green-vertical (RHGV) such that stimuli expected to look the same color were averaged. For instance, the first bar reflects testing with the vertical stimulus in subgroup RVGH averaged with the horizontal lines in subgroup RHGV, both of which should appear the same color (green when viewed with head tilted). The second bar reflects the reverse stimuli, the horizontal bars in subgroup RVGH and the vertical bars in subgroup RHGV. The third and fourth bars show the same conditions, except as viewed with the head upright.

As can be seen from the third and fourth bars, turning the head to upright during testing caused the colors to reverse. Stimuli that previously looked pink now looked green, and vice versa. Therefore, also as expected, induction with both a retinal and a spatial contingency led to a predominantly retinal aftereffect. The effect appeared smaller when tested in the upright position than when in 


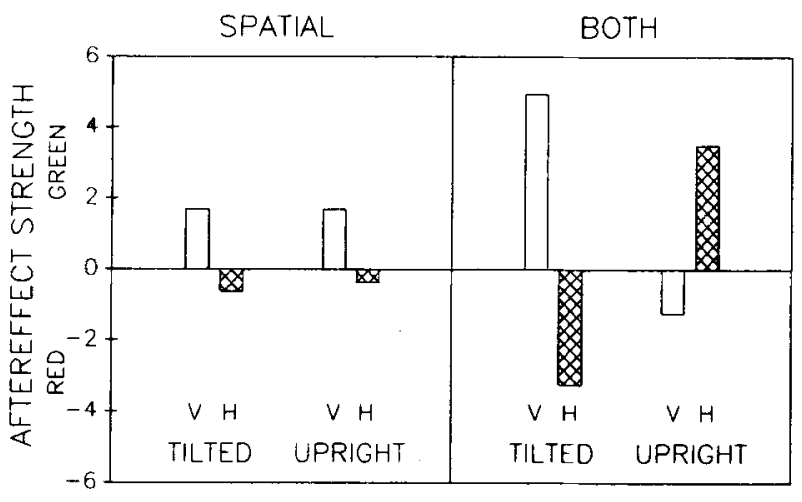

Figure 2. Mean color strength seen on achromatic gratings for the spatial-only group and the both-control group as a function of head position and stimulus orientation. $V=$ objectively vertical stimuli for the RVGH subgroup and objectively horizontal stimuli for the GVRH subgroup. $H=$ objectively horizontal stimuli for the RVGH subgroup and objectively vertical stimuli for the GVRH subgroup.

the tilted position; average strength at the noninduced head position was $58 \%$ that of the induced position. Because quantitative data on test displays viewed from different head positions is unavailable, whether the present decrement is standard cannot be determined. ${ }^{3}$ Part of the decrement might have occurred because the eyes were not fully rotated when the subject was tilted, both because of counterrotation of the eyes with head tilt and because it was difficult to ensure that the subjects were fully at $90^{\circ}$. This would imply that the same stimulus viewed upright and tilted would differ retinally by less than $90^{\circ}$, which would in turn be expected to produce less than complete color reversal. Of course, another explanation is that part of the decrement was due to a small spatial component, a hypothesis difficult to rule out at this point.

The data from the spatial-only group is shown in the left panel of Figure 2. It is apparent that, relative to induction with both contingencies, aftereffects here were minimal. There does appear to be a small spatial effect with the expected pattern of generalization for a spatial contingency: colors remained unchanged with head tilt. ${ }^{4}$ The average strength effect was $28 \%$ that of the control group (comparison made for tilted head position).

These observations were supported by analyses of variance (ANOVAs) performed separately on each group with head position (tilted, upright), color direction (RVGH, GVRH), stimulus orientation (vertical, horizontal), repetition (first, second), and line testing order (vertical first, horizontal first) as factors. We expected successful induction in the both-control group to be reflected by a triple interaction between orientation, color direction, and head position, which it was $[F(1,4)=54.58, p<.01]$. This shows that vertical and horizontal lines appeared different colors; which lines appeared red and which appeared green differed depending on which color direction was used in induction. In addition, everything reversed with head position. No other effect was significant (at .05 level of sig- nificance). In the spatial-only group, there was a significant orientation $\times$ color direction interaction $[F(1,4)=$ $33.11, p<.01$ ], suggesting there was an aftereffect in that group, which did not change with head tilt. There also might have been an unexpected color direction $\times$ head tilt interaction $[F(1,4)=9.00, p<.05] .{ }^{5}$ That is, patterns looked greener when viewed from the upright position than when viewed from the tilted position, but only for one of the color directions used in induction. There is no obvious explanation for this finding, but the difference was very small (mean $=-1.0$ when upright, -.50 when tilted). All remaining significant effects involved repetitions, which interacted with nearly everything. Analyses were repeated for just the first repetition for both groups, where no major differences were detected. Table 3 shows the predicted color direction as well as the actual color seen on the first and on the second repetition for each type of test trial.

To better compare the size of aftereffects independent of which color was seen, the data were transformed such that all "adaptive" colors seen, whether they were green or red, were coded as + , whereas all "nonadaptive" responses were coded as - . An ANOVA confirms results visible in the graph. A group (spatial, control) main effect $[F(1,8)=20.12, p<.01]$ confirms the greater strength in the control group. A main effect of head tilt $[F(1,8)=7.12, p<.05]$ reflects the overall greater strength while tested with the tilted head position. This is further clarified by the group $\times$ head tilt interaction $[F(1,8)=5.32, p=.05]$, which suggests that the group that was trained only while tilted, showed a decrement when tested upright, whereas the other group had equal strength for both head positions. The only other significant effect was a repetition interaction [line testing order $\times$ repetition, $F(1,8)=6.57, p<.05]$.

The subjects' verbal reports were also of interest. In the both-control group, 6 out of 8 subjects described the pattern in spatial terms, although the colors actually seen depended on the retinal relation. For instance, in the

Table 3

Experiment 1 Means

\begin{tabular}{|c|c|c|c|c|c|c|}
\hline \multirow[b]{2}{*}{$\begin{array}{l}\text { Test } \\
\text { Trial } \\
\end{array}$} & \multicolumn{3}{|c|}{ Spatial-Only Group } & \multicolumn{3}{|c|}{ Both-Control Group } \\
\hline & $\begin{array}{l}\text { Predicted } \\
\text { Color }\end{array}$ & Rep. 1 & Rep. 2 & $\begin{array}{c}\text { Predicted } \\
\text { Color }\end{array}$ & Rep. 1 & Rep. 2 \\
\hline & \multicolumn{3}{|c|}{ RVGH Subgroup } & \multicolumn{3}{|c|}{ RVGH Subgroup } \\
\hline $\begin{array}{l}\text { VT } \\
\text { VU } \\
\text { HT } \\
\text { HU }\end{array}$ & $\begin{array}{l}+ \\
+ \\
- \\
-\end{array}$ & $\begin{array}{l}+1.00 \\
+1.00 \\
-0.25 \\
-0.50\end{array}$ & $\begin{array}{l}+1.75 \\
+1.00 \\
-0.25 \\
-0.25\end{array}$ & $\begin{array}{l}+ \\
- \\
-\end{array}$ & $\begin{array}{l}+5.00 \\
-1.25 \\
-3.25 \\
+3.25\end{array}$ & $\begin{array}{l}+4.25 \\
-0.50 \\
-0.50 \\
+3.00\end{array}$ \\
\hline HU & \multicolumn{3}{|c|}{ GVRH Subgroup } & \multicolumn{3}{|c|}{ GVRH Subgroup } \\
\hline $\begin{array}{l}\text { VT } \\
\text { VU } \\
\text { HT } \\
\text { HU }\end{array}$ & $\begin{array}{l}- \\
- \\
+ \\
+\end{array}$ & $\begin{array}{l}-1.50 \\
-0.25 \\
+2.00 \\
+2.50\end{array}$ & $\begin{array}{l}-0.50 \\
-0.50 \\
+2.00 \\
+2.25\end{array}$ & $\begin{array}{l}- \\
+ \\
+ \\
-\end{array}$ & $\begin{array}{r}-4.50 \\
+4.50 \\
+5.75 \\
-0.50\end{array}$ & $\begin{array}{l}-4.75 \\
+3.25 \\
+4.75 \\
-2.75\end{array}$ \\
\hline
\end{tabular}

Note-Subgroups are described in physical coordinates. Each value is the mean of 4 subjects. $+=$ green, $-=$ red, $\mathrm{V}=$ vertical, $\mathrm{H}=$ horizontal, $\mathrm{T}=$ tilted head position, $\mathrm{U}=$ upright head position, $\mathrm{Rep}$. = repetition. 
GVRH subgroup, the pattern during induction was described as "green was always vertical and red was always horizontal," even though retinally it was the reverse. (The other 2 subjects were unable to describe any pattern.) In the spatial-only group, all $\mathbf{8}$ subjects correctly described the objective pattern (e.g., "vertical was green and red was sideways"), but the color aftereffect was minimal. These results provide another demonstration of the dissociation that can occur between the ME and subjects' conscious awareness. For instance, it has been found that the strength of the aftereffect is independent of whether the inducing stimuli occur inside or outside the focus of attention (Houck \& Hoffman, 1986).

Overall, the data suggest that it is relatively difficult to get color contingent on the objective orientation.

\section{EXPERIMENT 2}

To argue that the ME depends predominantly on retinal relationships requires that those retinal relations be sufficient to cause successful induction. When the standard ME is induced, color becomes contingent on retinal orientation. However, as noted earlier, a spatial contingency is available at the same time. Might the covariation of color and real-world orientation make possible the learning about retinal properties? The spatial regularity present in the standard ME may in some subtle way be a critical component of that phenomenon.

Whereas Experiment 1 removed the retinal contingency and left only the spatial contingency, Experiment 2 did the reverse and removed the spatial contingency instead. The purpose of Experiment 2 was to determine whether McCollough-like effects would occur when the only existing correlation was between color and retinal orientation. Consider a display that begins with red vertical lines followed by green horizontal lines, both viewed with the head upright. They are followed by different real-world stimuli, green vertical lines and red horizontal ones, except viewed with the head tilted $90^{\circ}$. The spatial correlation is zero: vertical lines are red only half the time and green the other half and likewise for horizontal lines. Yet there is a perfect retinal correlation: retinally vertical lines are always red, and retinally horizontal lines are always green. Retinally, it is equivalent to presenting green vertical and red horizontal stimuli with the head always tilted $90^{\circ}$, as presented in Experiment 1.

To complete the final cell of the experimental design, a group was run that received neither a retinal nor a spatial contingency. We expected that no systematic color would be seen on achromatic oriented lines during testing in this group. The manipulation can help identify some types of unplanned contingencies that result from the general paradigm. Whereas the control group in Experiment 1 set the upper limit on the expected size of an aftereffect, this control group set the lower limit. Vertical and horizontal grids were presented in the same color (e.g., green) while the subject was upright and the other color (e.g., red) while tilted.

\section{Method}

Subjects. The subjects were 16 female undergraduates participating for credit in introductory psychology at the University of Arizona.

Apparatus, Materials, and Procedure. Except where otherwise specified, the paradigm was the same as that of Experiment 1 . The subjects were randomly assigned to one of two groups: the retinalonly group or the neither-control group. Following initial testing and screening, the first two trials for the retinal-only group were a vertical and a horizontal display, one in red and the other in green, while the subject was in the upright position. After the subject switched into the tilted position, the colors of the vertical and horizontal lines also were switched such that spatially there was no relation between orientation and color but retinally there was (see Table 2). The direction of color-orientation pairs was counterbalanced. Subjects in the neither-control group saw the vertical and horizontal lines both in the same color while viewing from the upright position followed by vertical and horizontal lines in the other color while viewing from the tilted position. As in Experiment 1, the number of units of pink or green color matched to objectively achromatic displays served as the measure of chromatic aftereffects.

\section{Results and Discussion}

The data are shown in Figure 3. There is clearly a strong contingent aftereffect in the retinal-only group. Consequently, there need not be a relation between color and real-world orientation to produce the ME; a correlation between color and retinal orientation is sufficient. In addition, there is no evidence that the presence of a spatial contingency changes the $\mathrm{ME}$. If we compare the size of the effect when only a retinal contingency is present with the condition from Experiment 1 where both retinal and spatial contingencies were present, the values are close. When the head was tilted during testing, the green aftereffect had a strength of $5.25(S D=2.21)$ for the retinalonly group (Experiment 2$)$ and $4.94(S D=3.06)$ for the both-contol group (Experiment 1). The red aftereffect measured 3.88 $(S D=2.28)$ for the retinal-only group and 3.25 ( $S D=2.59$ ) for the both-control group. (It is more difficult to compare the values for the upright head position because one group [retinal-only] received training at that position whereas the other group did not.) To con-

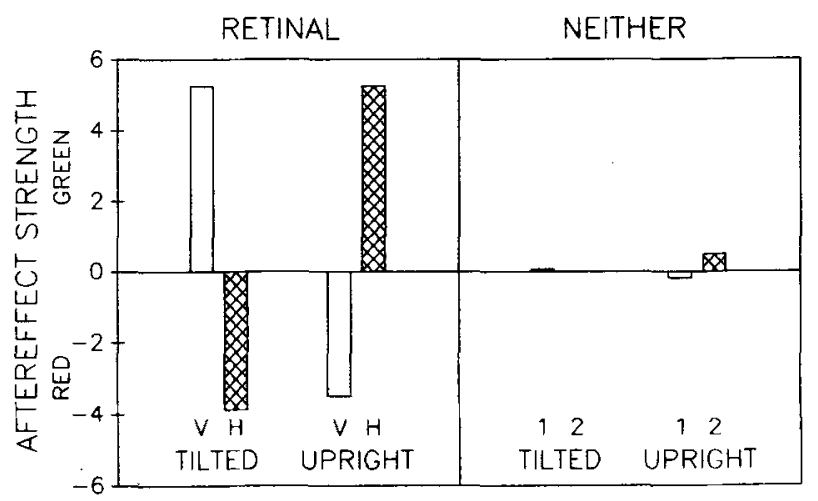

Figure 3. Mean color strength for the retinal-only and the neithercontrol groups. $V=$ vertical stimuli for the RVGH subgroup and horizontal stimuli for the GVRH subgroup. $H$ = horizontal stimuli for the RVGH subgroup and vertical stimuli for the GVRH subgroup. 1 = GURT subgroup; 2 = RUGT subgroup. 
clude definitively that the spatial contingency plays no role at all, either facilitatory or inhibitory, would require additional comparisons-including comparison with a group that received the standard ME with head upright during induction. Comparison of the retinal-only group with the spatial-only group shows the latter manipulation overall only $23 \%$ as effective as the former.

An ANOVA on the retinal-only group (same factors as in Experiment 1) verifies a reliable effect in that group [color direction $\times$ stimulus orientation $\times$ head tilt, $F(1,4)$ $=30.18, p<.01]$. The only other significant effect was an interaction between repetition and the above three factors [i.e., a four-way interaction; $F(1,4)=34.13, p<$ $.01]$, suggesting decline from the first to the second repetition.

The subjects' verbal reports suggest that most of the subjects in the retinal-only group ( 7 out of 8 ) were aware that the stimuli they received depended on the position of the head. The reports raise the issue that another contingency might have been influential. The retinal-only group actually had not only a retinal contingency but a complicated spatial one as well. There is a relation between color and objective orientation, if one considers the dependence on the third variable of head position. $\mathrm{Al}$ though it is logically possible that what looks like a retinal effect was instead due to a spatial effect contingent on head position, the possibility is remote. Because the subjects had difficulty learning a simple spatial contingency, as shown in Experiment 1, it is implausible that they would do better with a more complicated higher order spatial contingency. Where awareness and verbal reports seem dependent on real-world properties, actually seeing illusory colors seem to be caused by proximal stimulus patterns.

There was little systematic color aftereffect in the control group. It does appear that on the first repetition, all stimuli looked slightly green when the head was tilted and slightly pink when the head was upright; however, on the second repetition, that relation reversed [head tilt $\times$ repetition, $F(1,4)=24.00, p<.01]$. There is no obvious explanation, but the means are not large enough to be a concern. This was true of the other significant effects as well, all of which involved repetition or line testing order. Table 4 shows the predicted color direction, as well as the actual color seen, on the first and second repetition for each trial type. The data do not identify any particular filtering of only some types of trials, suggesting that the subjects did receive approximately the intended contingencies.

Note that, while the control stimuli removed all relations between color and orientation, they did introduce a systematic relation between color and head position. If color can become contingent on head position, all lines should look one color when viewed from the upright position and another when viewed from the tilted position. No statistical evidence was found that the contingency was learned [color direction $\times$ head position, $F(1,4)<1$ ] Figure 3 (right panel) shows the data for this group collapsed across stimulus orientation. The first column shows color appearance for the green-upright/red-tilted (GURT) subgroup while viewing from the tilted head position (which should appear green), the second column shows color appearance for the RUGT subgroup while viewing from the tilted position (which should appear red), the third and fourth columns show color appearance for the same subgroups while viewing from the upright position (which should appear red and green, respectively). If there is a trend in the correct direction, it is minimal.

The main conclusion from Experiment 2 is that a retinalonly contingency, unlike a spatial-only contingency, is highly effective at producing McCollough-like effects.

\section{EXPERIMENT 3}

The purpose of Experiment 3 was to investigate further the apparent spatial ME found in Experiment 1. Although the illusory colors seen were small, they did appear to be entirely created by, and under the control of, the real-world or spatial orientation of the stimulus. The effect could not have been produced by a simple hidden

Table 4

Experiment 2 Means

\begin{tabular}{|c|c|c|c|c|c|c|}
\hline \multirow[b]{2}{*}{$\begin{array}{l}\text { Test } \\
\text { Trial }\end{array}$} & \multicolumn{3}{|c|}{ Retinal-Only Group } & \multicolumn{3}{|c|}{ Neither-Control Group } \\
\hline & $\begin{array}{l}\text { Predicted } \\
\text { Color }\end{array}$ & Rep. 1 & Rep. 2 & $\begin{array}{c}\text { Predicted } \\
\text { Color }\end{array}$ & Rep. 1 & Rep. 2 \\
\hline & \multicolumn{3}{|c|}{ U-RVGH/T-RHGV Subgroup } & \multicolumn{3}{|c|}{ RUGT Subgroup } \\
\hline $\begin{array}{l}\text { VT } \\
\text { VU } \\
\text { HT } \\
\text { HU }\end{array}$ & $\begin{array}{l}- \\
+ \\
+ \\
-\end{array}$ & $\begin{array}{r}-4.25 \\
+5.75 \\
+5.25 \\
-4.00\end{array}$ & $\begin{array}{l}-3.25 \\
+4.50 \\
+4.00 \\
-2.75\end{array}$ & $\begin{array}{l}- \\
+ \\
- \\
+\end{array}$ & $\begin{array}{l}-0.50 \\
+0.25 \\
+0.25 \\
+1.50\end{array}$ & $\begin{array}{l}+0.50 \\
-0.25 \\
-0.25 \\
+0.50\end{array}$ \\
\hline & \multicolumn{3}{|c|}{ U-GVRH/T-GHRV Subgroup } & \multicolumn{3}{|c|}{ GURT Subgroup } \\
\hline $\begin{array}{l}\text { VT } \\
\text { VU } \\
\text { HT } \\
\text { HU }\end{array}$ & $\begin{array}{l}- \\
- \\
+\end{array}$ & $\begin{array}{l}+6.50 \\
-4.00 \\
-4.25 \\
+5.75\end{array}$ & $\begin{array}{l}+5.25 \\
-3.25 \\
-3.75 \\
+5.00\end{array}$ & $\begin{array}{l}+ \\
- \\
+ \\
-\end{array}$ & $\begin{array}{r}-0.25 \\
+0.25 \\
0.00 \\
-0.50\end{array}$ & $\begin{array}{r}+0.25 \\
0.00 \\
+0.25 \\
-0.50\end{array}$ \\
\hline
\end{tabular}

Note-Subgroups are described in physical coordinates. Each value is the mean of 4 subjects. $+=$ green, $-=$ red, $\mathrm{V}=$ vertical, $\mathrm{H}=$ horizontal, $\mathrm{T}=$ tilted head position, $\mathrm{U}=$ upright head position, Rep. = repetition. 
retinal contingency, because the colors appeared identical for orthogonal head positions (see Note 4). However, there is a more complicated retinal contingency that might produce aftereffects that only appear to be spatial.

Counterrotation of the eyes will prevent the retinal orientation from changing the full $90^{\circ}$ when the head is tilted $90^{\circ}$. Consequently, vertical and horizontal lines do not completely trade places retinally with head tilt. This produces two additional stimuli, near vertical and near horizontal, and it is a pattern involving all four stimuli that subjects actually receive in training. Consider an example where red-vertical and green-horizontal pairs are viewed in a upright position. Tilting the head produces red-near horizontal and green-near vertical, rather than red-horizontal and green-vertical. Strictly speaking, there is a correlation between retinal orientation and color. If all four stimuli could be accommodated, then retinally vertical lines would appear green, near-vertical lines would appear red, horizontal lines would appear red, and nearhorizontal lines would appear green. If this complicated retinal relation were learned, when objectively vertical and horizontal lines were viewed with the head tilted the colors would appear to remain the same as when upright.

A second alternative involves the hypothesis that contingent aftereffects can themselves become contingent on head position. When the head is upright, red vertical and green horizontal lines are paired; however, when the head is tilted, it is green-horizontal and red-vertical instead. The results of the Experiment 2 control group suggested that color by itself did not become contingent on head position, but it is not clear whether a color-orientation bond could do so. Finally, a related alternative is a higher order effect contingent not on head position but on some retinal property present in the situation, such as location. When a subject tilted $90^{\circ}$ toward the right, the stimuli were retinally displaced upward and to the left. The two opposite contingencies might have become dependent on those contextual features rather than canceling one another.

We tested the first and, to some extent, the third of these alternatives to a genuine spatial ME, both of which are based on complicated retinal relations. The second alternative, based on head positions, is more difficult to tease apart and will be left for future explorations. We simulated the complicated retinal regularities to determine if they could, in and of themselves, produce any aftereffects. All training and testing took place with the subjects in the upright head position; the subjects were shown four distinct stimuli that closely matched what would have been generated on the retina under both head positions in Experiment 1. For instance, following red vertical and green horizontal lines, the subjects saw red $105^{\circ}$ (near-horizontal) and green $15^{\circ}$ (near-vertical) lines. The latter two stimuli were presented slightly farther to the left than were the first two in order to simulate part of the position change that occurred with head tilt. A difference of $15^{\circ}$ was the estimated deviation from vertical and horizontal in the prior experiment, due both to counterrotation and to the fact that the subjects tended to be physically tilted less than $90^{\circ}$ because of neck strain. If training were successful, achromatic vertical lines would appear green, but the nearby $15^{\circ}$ lines would appear red. Horizontal lines would also appear red, and $105^{\circ}$ lines would appear green.

How far apart would vertical and near-vertical lines need to be before they could be successfully induced with complementary colors? Initial findings (Fidell, 1970) suggested that the oriented stimuli need to be greater than $15^{\circ}$ apart, but subsequent research suggested that a separation as small as $11^{\circ}$ or $12^{\circ}$ could suffice (see Stromeyer, 1978). However, most experiments on the ME use only two inducing stimuli; consequently, the properties of a more complicated four-stimulus induction are unknown.

A second group of subjects received only the $15^{\circ}$ and $105^{\circ}$ stimuli to ensure those new stimuli were effective in this paradigm. This also allowed assessment of the size of any effect that might occur in the group receiving four stimuli, which differed from this group by additionally receiving trials at the two other orientations $\left(0^{\circ}\right.$ and $\left.90^{\circ}\right)$.

\section{Method}

Subjects. The subjects were 16 female undergraduates participating for credit in introductory psychology at the University of Arizona.

Apparatus and Materials. Only the $0^{\circ}$ headrest was used. Two additional types of stimuli were generated for this experiment: gratings oriented at $15^{\circ}$ (near vertical) and gratings orientated at $105^{\circ}$ (near horizontal). The former orientation was what would occur if the subects looked at horizontal gratings while their eyes were rotated a total of $75^{\circ}$ clockwise (i.e., $15^{\circ}$ less than the intended $90^{\circ}$ ), and the latter while looking at vertical gratings under the same rotation. The lines that made up the $15^{\circ}$ and $105^{\circ}$ stimuli were slightly jagged, but this was not expected to interfere with induction. All displays were made smaller to enable the tilted patterns to fit on the screen. This was accomplished by eliminating and shortening bars but keeping the spatial frequencies the same as in the previous experiments. The tilted patterns were always presented 1.5 in. to the left of where the vertical and horizontal patterns were presented to simulate part of the change in retinal position that would have occurred when the subjects were tilted to the right. The up/down position could not be changed due to limited space on the monitor.

Procedure. The subjects were randomly assigned to one of two groups: the four-stimuli simulation group or the two-stimuli control group.

Initial screening and testing. The procedure was the same as in the previous experiments, except the vertical and horizontal patterns previously viewed from the tilted $90^{\circ}$ position were replaced with the tilted patterns and viewed from the upright position. Thus, the second and fourth trials consisted of $105^{\circ}$ in the upper left and lower right quadrants and $15^{\circ}$ in the upper right and lower left. Each stimulus was a $4 \times 4$ in. square patch.

Induction. The sequence of the four stimuli in the four-stimulus simulation group were red- $0^{\circ}$ (vertical), green- $90^{\circ}$ (horizontal), red- $105^{\circ}$ (near horizontal), and green- $15^{\circ}$ (near vertical) for half of the subjects. The other half received the opposite color-orientation pairings. In the two-stimulus control group, only the third and fourth patterns were presented, and the first and second stimuli were replaced with a blank (black) screen. The subjects in the control group therefore received exactly the same number of near-horizontal and near-vertical trials as did the experimental group, and with the 
same temporal patterns. Each stimulus was a $5 \times 5$ in. square patch. The subjects were instructed to push the joystick button after hearing the beep following each pair of stimuli, in order to match the procedure to the previous experiments.

Test. As in initial testing and screening, trials from the previous experiments where the subjects matched to vertical or horizontal lines while they were tilted were replaced with trials with the nearvertical and near-horizontal lines while upright. Thus, in four of the eight trials, the subjects matched to $15^{\circ}$ and $105^{\circ}$ stimuli; in the other four trials, they matched to $0^{\circ}$ and $90^{\circ}$ stimuli.

\section{Results and Discussion}

The right panel of Figure 4 shows the outcome for the two-stimulus control group, for which color clearly became contingent on orientation. As expected, induction with the orthogonal gratings of $15^{\circ}$ and $105^{\circ}$ did produce contingent aftereffects (first and second bars in the right panel). The aftereffect was weaker than that produced by the $0^{\circ}$ and $90^{\circ}$ stimuli in Experiment 1 (both-control group), which is not surprising because half as many training trials were used in Experiment 3. Colors were also seen on the noninduced vertical and horizontal stimuli (third and fourth bars in right panel), where training at $15^{\circ}$ transferred to the nearby $0^{\circ}$, and $105^{\circ}$ transferred to $90^{\circ}$ (see Table 5). The data were entered in a two-way ANOVA with color direction (two levels: red $-15^{\circ} /$ green $-105^{\circ}$; green- $15^{\circ} / \mathrm{red}-105^{\circ}$ ) and stimulus orientation (four levels: $0^{\circ}, 15^{\circ}, 90^{\circ}, 105^{\circ}$ ) as factors. As expected, the color direction $\times$ stimulus orientation interaction was significant $[F(3,18)=24.06, p<.001]$. No other effect was significant.

In the four-stimuli simulation group, adding trials at $0^{\circ}$ and $90^{\circ}$ to those at $15^{\circ}$ and $105^{\circ}$ in a fashion that went opposite to the natural pattern of transfer reduced the size of the effect (see Figure 4, left panel). Yet aftereffects might not have been completely eliminated [color direction $\times$ stimulus orientation, $F(3,18)=3.50, p<.05$; see Note 5]. The colors that were seen reflect the relationship presented accurately, where nearby stimulus orientations (e.g., $15^{\circ}$ and $0^{\circ}$, second and third bars) ap-

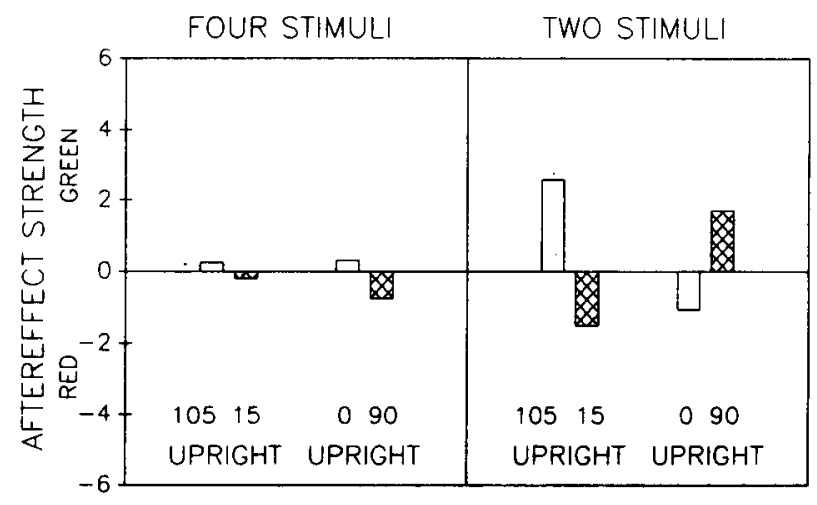

Figure 4. Mean color strength for the four-stimuli simulation group and the two-stimuli control.

pear as opposite colors. To facilitate comparison to the spatial-only group of Experiment 1, the data are shown in the same order as that group (see Figure 2, left panel). The head-tilt trials are replaced with the corresponding retinally simulated trials. The data suggest that what appeared to be a spatial ME in Experiment 1 could have instead been under the control of complicated retinal properties.

The four induction stimuli might have led to two independent MEs that became contingent on how far left or right the patterns were. Alternatively, all four stimuli might have been accommodated within a single McCollough-like effect, which would suggest that contingent aftereffects can adhere to more complex relations between orientation and color than was previously thought. The present design did not allow these two alternatives to be distinguished. Note that these effects are of interest in their own right and will be the subject of future investigations. We also cannot conclude definitively that the results in. Experiment 3 depended on retinal rather than spatial properties, although we suspect they were, given the previous experiments. Verification would require that the illusory colors reversed with the head tilted, which may require a paradigm more

Table 5

Experiment 3 Means

\begin{tabular}{|c|c|c|c|c|c|c|}
\hline \multirow[b]{2}{*}{$\begin{array}{l}\text { Test } \\
\text { Trial } \\
\end{array}$} & \multicolumn{3}{|c|}{ Four-Stimuli Group } & \multicolumn{3}{|c|}{ Two-Stimuli Group } \\
\hline & $\begin{array}{c}\text { Predicted } \\
\text { Color }\end{array}$ & Rep. 1 & Rep. 2 & $\begin{array}{c}\text { Predicted } \\
\text { Color }\end{array}$ & Rep. 1 & Rep. 2 \\
\hline & \multicolumn{3}{|c|}{$\mathrm{R} 105^{\circ} \mathrm{G} 15^{\circ} / \mathrm{R} 0^{\circ} \mathrm{G} 90^{\circ}$ Subgroup } & \multicolumn{3}{|c|}{$\mathrm{R} 105^{\circ} \mathrm{G} 15^{\circ}$ Subgroup } \\
\hline $\begin{array}{r}105^{\circ} \\
0^{\circ} \\
15^{\circ} \\
90^{\circ}\end{array}$ & $\begin{array}{l}+ \\
+ \\
- \\
-\end{array}$ & $\begin{array}{l}-0.25 \\
-0.50 \\
-0.25 \\
-0.75\end{array}$ & $\begin{array}{r}-0.25 \\
+0.75 \\
0.00 \\
-1.25\end{array}$ & $\begin{array}{l}+ \\
-* \\
- \\
+^{*}\end{array}$ & $\begin{array}{l}+3.00 \\
-0.50 \\
-2.25 \\
+2.50\end{array}$ & $\begin{array}{l}+3.25 \\
-0.50 \\
-1.25 \\
+2.25\end{array}$ \\
\hline $90^{\circ}$ & \multicolumn{3}{|c|}{$\mathrm{G} 105^{\circ} \mathrm{R} 15^{\circ} / \mathrm{G} 0^{\circ} \mathrm{R} 90^{\circ}$ Subgroup } & \multicolumn{3}{|c|}{$\mathrm{G} 105^{\circ} \mathrm{R} 15^{\circ}$ Subgroup } \\
\hline $\begin{array}{r}105^{\circ} \\
0^{\circ} \\
15^{\circ} \\
90^{\circ}\end{array}$ & $\begin{array}{l}- \\
- \\
+ \\
+\end{array}$ & $\begin{array}{l}-0.25 \\
-0.75 \\
+1.00 \\
+0.25\end{array}$ & $\begin{array}{l}-0.25 \\
-0.25 \\
+0.50 \\
+0.75\end{array}$ & $\begin{array}{l}- \\
+* \\
+ \\
-*\end{array}$ & $\begin{array}{l}-1.25 \\
+0.75 \\
+2.75 \\
-2.25\end{array}$ & $\begin{array}{l}-1.00 \\
+1.25 \\
+1.25 \\
-1.00\end{array}$ \\
\hline
\end{tabular}

Note-Subgroups are described in physical coordinates. Each value is the mean of 4 subjects. $+=$ green,

$-=$ red. *Assuming generalization from the nearest orientation used in induction. 
suited to measuring very small effects, such as psychometric function shifts (Allan, Siegel, Toppan, \& Lockhead, 1991).

Can all of the apparent spatial ME be accounted for by retinal "artifacts"? Comparison of the simulation group in Experiment 3 with the spatial-only group of Experiment 1 shows that the spatial-only group does have a larger overall aftereffect [mean $=1.09, S D=.57$; fourstimulus simulation group mean $=.38, S D=.47 ; t(14)$ $=2.77, p<.025]$. However, Experiment 3 could not simulate all the hypothesized retinal relations present, such as those based on head position. Further investigation is needed, also using the paradigms better suited to comparing very small effects.

While this experiment raises a number of new questions, overall the data suggests that at least part of the already small spatial effect may be due instead to hidden retinal properties.

\section{GENERAL DISCUSSION}

The outcome of the present study can be summarized as follows. When there is a statistical relationship between the color of bars and only the orientation of those bars on the retina, color does become contingent on orientation. The resulting illusory colors are as strong as when the real-world or spatial orientation of the bars is equally correlated with color. This suggests that retinal properties are sufficient for successful induction and that regularities involving the real world are not a necessary part of the ME. In addition, isolating instead the linkage between real-world orientation and color led to a relatively small contingent aftereffect, at least part of which might have been due to complex hidden retinal relationships.

Is there any clear evidence for a spatial ME? Studies in which both retinal and spatial contingencies are present during induction and then dissociated during testing have produced conflicting results. Harris $(1970,1980)$ made color contingent on both spatial and retinal spatial frequency by alternating green wide bars and red narrow bars for several minutes. During testing, he assessed the neutral point for a variety of different spatial frequencies by having subjects adjust the distance of the stimuli until they appeared neither green nor pink. He found that subjects set the distance of each stimulus such that the retinal spacing of all the different gratings was identical, suggesting there was no influence from the actual or the perceived bar widths.

On the other hand, Jordan and Uhlarik (1983), using similar induction conditions, found that apparent spatial frequency did influence the position of the neutral point. During testing, they placed their stimuli either in an upper region or in a lower region of a picture of a receding corridor. Within the pictorial corridor, two gratings of exactly the same retinal spatial frequency appeared to be different widths. They found that the neutral point was always set about $1 / 4$ of a meter closer for the stimuli placed in the lower region, for a variety of different spatial fre- quencies. Although the percentage influence of apparent spatial frequency cannot be calculated, ${ }^{6}$ it was greater than the $0 \%$ reported by Harris.

Resolution of the conflicting data may involve the opposite biases of the two testing procedures. The critical comparisons in Jordan and Uhlarik's experiment involved changing the apparent spatial frequency while keeping the retinal spatial frequency constant; for Harris, ${ }^{7}$ the reverse was true. In addition, there are at least two different ways to uncouple coordinates: entirely stimulus-based changes and changes that affect the observer or the relationship between the observer and the stimulus. Embedding the stimuli within a picture of a corridor is clearly an instance of the former; changing the distance between the grating and the observer may use the latter. The effectiveness of apparent properties may differ depending on which mechanisms involved in size perception and size constancy are tapped: mechanisms based entirely on information contained within the stimulus or mechanisms that compute the distance of the stimulus from an observer. The present paradigm makes changes to the observer to dissociate the coordinates; it is possible that changes to the stimulus can produce stronger spatial MEs. ${ }^{8}$

Conflicting results have also been found when color has been made contingent on orientation. In a careful quantitative study, Ellis (1976) induced subjects (who were tilted $50^{\circ}$ ) with physically vertical and horizontal lines of complementary colors. He also photographed eye position to get an exact measure of the retinal orientation of the stimuli. During testing, subjects were seated upright and had to change the orientation of a grating until it appeared to produce the strongest color. Considering one of the stimuli, if color had become contingent on apparent orientation, then the grating would be set to $0^{\circ}$ (vertical). If instead color had become contingent on retinal orientation, then the grating would be set to $46^{\circ}$, the difference between the objective position of the stimulus and the actual position of the eyes during induction. Subjects set the grating to an average of $45.5^{\circ}$, remarkably close to the prediction of $46^{\circ}$. No evidence was found that the ME is anything but retinally locked, at least when both contingencies are present during induction.

However, Mikaelian (1976) came to exactly the opposite conclusion using a complicated and intriguing means to dissociate the coordinates. Unfortunately, his procedure may not have been successful. He first adapted subjects to tilting prisms before he induced the ME. Thus, during induction, color could have become contingent on either the retinal orientation (e.g., $79^{\circ}$ ) or the apparent orientation (e.g., $69^{\circ}$ ), which was made different by having adapted to the tilting prisms. To determine which occurred, he used a test procedure similar to that of Ellis and compared the orientation of maximum color chosen first with, and then without, looking through the prism. This is problematic because those two values should differ by exactly the amount of the prism distortion, regardless of which coordinates were influential-unless decay happened to occur between the two tests. It would be desir- 
able to repeat some version of this experiment, especially since it attempts to pull apart retinal and spatial coordinates in yet a third way. Rather than immediate changes to the stimulus (used by Jordan \& Uhlarik, 1983), or the relationship between stimulus and observer (used in the present study, and by Ellis, 1976, and arguably by Harris, 1970,1980 ), it uses perceptual learning. It would be interesting if under these conditions apparent properties can play the largest role.

Interesting issues for future investigations include elucidating the conditions under which spatial properties are influential and determining if that effect adheres to the same basic rules as the ME. However, the main conclusion is that the ME is predominantly retinal. It was known before that when both contingencies are present, retinal properties dominate. The present study makes it plain that even when the only available relationship involves real-world properties, learning about those properties is still minimal. Consequently, the processes underlying the ME are not concerned with real-world properties of objects or events.

Returning to the issue raised in the Introduction, should such a lack of concern for the real world rule out explanations of the ME based on learning? The answer now depends on what one means by "learning." Some researchers of the ME, particularly those who advocate a Pavlovian interpretation, use the terms "learning," "Pavlovian conditioning," and "associative learning" interchangeably (e.g., Siegel et al., 1992). Yet not all learning accounts of the ME need be Pavlovian conditioning (e.g., Savoy, 1984; Skowbo, 1984). If one adopts instead a broad view of learning, then the present outcome does not rule out all learning-based explanations, but it does make some classes of explanations seem less sensible. ${ }^{9}$

Any account in which the goal is to apprehend new information about the world would be ill served by a process that is insensitive to that information. Consequently, Pavlovian conditioning may become a less attractive candidate. In the last 20 years, conditioning has been understood to be a process that apprehends new relations among events in the world, at least by some theoreticians (e.g., Rescorla, 1988; Rescorla \& Holland, 1976). Rescorla and Holland (p. 172) explain that "we view conditioning more as a way in which the organism learns about the causal relations in his environment." More recently, Rescorla summarizes that "conditioning is now described as the learning of relations among events so as to allow the organism to represent its environment" (p.151) and that "such learning is a primary means by which the organism represents the structure of its world" (p. 152). In general, any process that is about apprehending new information from the world may not be applicable. What type of learning remains?

What remains is perceptual learning, the goal of which is to correct internal malfunctions or otherwise improve the ability to perceive rather than to learn about the world (see Bedford, 1989, 1992, 1993a, 1993b). Perceptual learning may be generally described as occurring whenever the same proximal stimulus comes to lead to a different percept and continues to do so in the absence of new information. Such processes would likely need to operate on retinal properties. This general framework is consistent with the views of the handful of researchers (Bedford, 1992, 1993b; Dodwell \& Humphrey, 1990; Held, 1980; Warren, 1985) who explicitly suggest ME reflects self-correction. All four interpretations share the view that stimuli provide information that something internal is not functioning optimally. For Held, colors on oriented edges suggest an optical defect, such as misalignment of the optical axis of the lens from the fixation axis. Warren suggests that repeated stimulation can keep perceptual systems optimal by shifting the underlying bases for judgments toward the previously encountered stimulation. Dodwell and Humphrey suggest that there should not be a long-term statistical correlation between color and orientation, and the presence of one suggests the need for an internal "error correcting device." Recently, Bedford suggested that the instigation for error correction is the detection that a single object is changing color when it is rotated with respect to the observer, which violates preexisting constraints about the nature of objects.

While these views are distinct from traditional learning, they are also far removed from "fatigue" models, the usually described alternative to traditional learning views. Whereas fatigue models tend to imply the $\mathrm{ME}$ is an unavoidable by-product of human machinery, the perceptual learning views instead suggest active intelligent calibrations that serve useful functions. Rather than traditional learning models or fatigue models, error correction provides a third class of models that may prove the best framework for future investigations.

\section{REFERENCES}

Allan, L. G., \& Siegel, S. (1986). McCollough effects as conditioned responses: Reply to Skowbo. Psychological Bulletin, 100, 388-393. Allan, L. G., Siegel, S., Toppan, P., \& Lockhead, G. R. (1991). Assessment of the McCollough effect by a shift in psychometric function. Bulletin of the Psychonomic Society, 29, 21-24.

BEDFORD, F. L. (1989). Constraints on learning new mappings between perceptual dimensions. Journal of Experimental Psychology: Human Perception \& Performance, 15, 232-248.

BEDFORD, F. L. (1992). Constraints on perceptual learning: Objects and dimensions. Manuscript submitted for publication.

BeDFord, F. L. (1993a). Perceptual and cognitive spatial learning. Journal of Experimental Psychology: Human Perception \& Pefformance, 17, 517-530.

Bedford, F. L. (1993b). Perceptual learning. In D. Medin (Ed.), Psychology of learning \& motivation, 30, 1-60.

Broerse, J., \& Crassini, B. (1981). Comments on the use of perceptually ambiguous figures as McCollough-effect stimuli. Perception \& Psychophysics, 30, 399-402.

Broerse, J., \& CRASSINI, B. (1986). Making ambiguous displays unambiguous: The influence of real colors and colored aftereffects on perceptual alternation. Perception \& Psychophysics, 39, $105-116$.

Day, R. H., Webster, W. R., Gillies, O., \& CRAssini, B. (1992). Spatial-frequency-contingent color aftereffects: Adaptation with onedimensional stimuli. Perception \& Psychophysics, 51, 57-65. 
Dodwell, P. C., Humphrey, G. K. (1990). A functional theory of the McCollough effect. Psychological Revien, 97. 78-89.

Ellis, S. R. (1976). Orientation constancy of the McCollough effect Perception \& Psychophysics, 19, 183-192.

Fidell, L. S. (1970). Orientation specificity in chromatic adaptation of human "edge-detectors." Perception \& Psychophysics, 8. 235-237

GIBSON, A., HARrIs, C. S. (1968, August). The Mc Collough effect. Color adaptation of edge-detectors or negative afierimages? Paper presented at Eastern Psychological Association. Washington. DC.

HARRis, C. S. (1970). Effect of viewing distance on a color aftereffect specific to spatial frequency. Psychonomic Science, 21, 350. (Abstract)

HARRIS, C. S. (1980). Insight or out of sight?: Two examples of perceptual plasticity in the human adult. In C. S. Harris (Ed.). Visual coding and adaptability (pp. 95-149). Hillsdale, NJ: Erlbaum.

Harris, C. S., Gibson, A. R. (1968). Is orientation-specific color adaptation in human vision due to edge detectors, afterimages, or "dipoles"'? Science, 162, 1506-1507.

HELD, R. (1980). The rediscovery of adaptability in the visual system: Effects of intrinsic and extrinsic chromatic dispersion. In C. S. Harris (Ed.), Visual coding and adaprability (pp. 69-94). Hillsdale, NJ: Erlbaum.

Houck, M. R., \& Hoffman, J. E. (1986). Conjunction of color and form without attention: Evidence from an orientation-contingent color aftereffect. Journal of Experimental Psychology: Human Perception \& Performance, 12, 186-199.

Jenkins, B., Ross, J. (1977). McCollough effect depends upon perceived organization. Perception, 6, 399-400.

JoRDAN, K., \& UHLARIK, J. (1983), Contextual influences on a spatialfrequency-contingent color aftereffect. Perception \& Psychophysics, $33,420-424$.

MAyHEW, J. E. W., \& ANSTIS, S. M. (1972). Movement aftereffects contingent on color, intensity, and pattern. Perception \& Psychophysics, 12, 77-85.

McCollough, C. (1965). Color adaptation of edge detectors in the human visual system. Science, 149, 1115-1116.

Meyer, G. E., \& Philuips, D. (1980). Faces, vases, subjective contours, and the McCollough effect. Perception, 9, 603-606.

Mikaelian, H. H. (1976). Plasticity of orientation specific chromatic aftereffects. Vision Research, 16, 459-462.

MurCH, G. M. (1976). Classical conditioning of the McCollough effect: Temporal parameters. Vision Research, 16, 615-619.

Rescorla, R. A. (1988). Pavlovian conditioning: It's not what you think it is. American Psychologist, 43, 151-160.

Rescorla, R. A., Holland, P. C. (1976). Some behavioral approaches to the study of learning. In M. R. Rosenzweig \& E. L. Bennett (Eds.), Neural mechanisms of learning and memory (pp. 165192). Cambridge, MA: MIT Press.

SAvoY, R. L. (1984). "Extinction" of the McCollough effect does not transfer interocularly. Perception \& Psychophysics, 36, 571-576.

Siegel, S., \& AlLAN, L. G. (1987). Contingency and the McCollough effect. Perception \& Psychophysics, 42, 281-285.

Siegel, S., Allan, L. G., Roberts, L., \& Eissenberg, T. (1990). Spatial contingency and the McCollough effect. Perception \& Psychophysics, 48, 307-312.

Siegel, S., Allan, L. G., Eissenberg, T. (1992). The associative basis of contingent color aftereffect. Joumal of Experimental Psychology: General, 121, 79-94.

Skowвo, D. (1984). Are McCollough effects conditioned responses? Psychological Bulletin, 34, 552-554.

SKowBo, D., * ForSTER, T. (1983). Further evidence against a classical conditioning model of McCollough effects. Perception \& Psychophysics, 34, 552-554.

STROMEYER, C. F. (1978). Form-color aftereffects in human vision. In R. Held, H. Leibowitz, \& H. L. Teuber (Eds.), Handbook of sensory physiology: Vol. 8. Perception (pp. 97-142). New York: Springer-Verlag.
Stromeyer. C. F. . Mansheid. R. J. W. (1970). Colored aftereffects produced with moving edges. Perception \& Psychophysics, 7, 108-114.

Thompson, P., LATCHFord, G. (1986). Colour-contingent after-effects are really wavelength-contingent. Nature, 320,525-526.

Uhtarik. J.. Pringt.F, R., BrigetL.. M. (1977). Color aftereffects contingent on perceptual organization. Perception \& Psychophysics. 22. $506-510$

WARREN. R. (1985). Criterion shift rule and perceptual homeostasis. Psychological Review. 92, 574-584.

WeBSTER, W. R., DAY, R. H. \& WILLENBERG, K. (1988). Orientationcontingent color aftereffects are determined by real color, not induced color. Perception \& Psychophysics, 44, 43-49.

WESTBROOK, R. F., \& HARRISON, W. (1984). Associative blocking of the McCollough Effect. Quarterly Journal of Experimental Psychology, 36A, 309-318.

WyszeckI, G. (1986). Color appearance. In K. R. Boff, L. Kaufman, \& J. P. Thomas (Eds.), Handbook of perception and human performance: Vol I. Sensory processes \& perception (pp. 9-1 to 9-57). New York: Wiley

\section{NOTES}

1. The term spatial orientation will be used interchangeably with both the apparent or perceived orientation and the physical or real-world orientation, which are assumed to be identical in this study.

2. This value was chosen on the basis of pilot data that indicated that an aftereffect under optimal conditions was $\mathbf{8}$ units, twice the chosen cutoff.

3. The subjects were always tested in a tilted position for the first trial, but this cannot by itself account for the decrement. The other groups in this experiment, as well as subsequent experiments, were also tested first while tilted but no decrement was observed.

4. The effect cannot be due to a simple retinal aftereffect where the subjects systematicaily filtered out all trials received while they were tilted. This scenario would produce opposite colors for orthogonal head positions.

5. If the criterion for statistical significance is adjusted for using multiple ANOVAs, then this effect is only marginally significant. Future research is needed to determine if it is a genuine effect.

6 . The difference in perceived spatial frequency produced by the corridor was not measured in the study.

7. It is actually not clear how the subjects would have completed the task had color become contingent on apparent rather than retinal spatial frequency. Since the apparent spatial frequency would not change as a function of distance, whatever color was seen should remain unchanged by distance. Under these circumstances, it would therefore seem difficult to find a null point at all.

8. Possibly relevant is the claim that perceived organization can influence the ME when ambiguous stimuli are used for testing (Jenkins \& Ross, 1977; Meyer \& Phillips, 1980; Uhlarik, Pringle, \& Brigell, 1977; but see also Broerse \& Crassini, 1981, 1986, for alternative accounts of the same data). Like those of Jordan and Uhlarik, these procedures manipulate the stimuli to demonstrate an effect of apparent properties.

9. We are not claiming that the our results prove or disprove any theory, but rather that some classes of theories more naturally accommodate insensitivity to environmental properties and sensitivity to retinal properties. Any single issue relevant to the ME has led to cogent arguments on different sides of the debate (e.g., stimuli capable of inducing the $\mathrm{ME}$ and the effects of contingency manipulations), and it is likely that a number of issues taken together are needed

(Manuscript received April 19, 1992; revision accepted for publication March 11, 1993.) 